\title{
Reconocimiento y clasificación de vehículos implementando un acelerómetro digital
}

\author{
Marco Antonio Jasso-Juárez ${ }^{1}$, Ignacio Hernández-Bautista ${ }^{2}$, \\ Juan José Carbajal-Hernández ${ }^{3}$, Juan Francisco Mosiño ${ }^{1}$, Raúl Santiago-Montero ${ }^{1}$ \\ ${ }^{1}$ Instituto Tecnológico de León, División de Estudios de Posgrados e Investigación, México \\ ${ }^{2}$ Cátedra CONACYT - Instituto Tecnológico de León, División de Estudios de Posgrados e \\ Investigación, México \\ ${ }^{3}$ Centro de Investigación en Computación, Instituto Politécnico Nacional, México

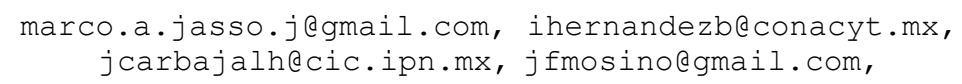

Resumen. En la actualidad, la contaminación por vibraciones urbanas es un problema creciente en las grandes ciudades. Este trabajo propone la creación de un sistema de análisis de las vibraciones producidas por automotores sobre avenidas urbanas. Mediante el procesamiento digital de señales provenientes de mediciones en el suelo, se presenta un método computacional que permite identificar diversas características como la magnitud, frecuencia y tipo de automotor. Resultados experimentales muestran evidencia un buen funcionamiento del sistema, proporcionando una herramienta que permitirá abordar estudios a mayor profundidad sobre los problemas que generan las vibraciones en vías de comunicación, así como su efecto en edificaciones circundantes.

Palabras clave: acelerómetro, vibraciones, reconocimiento de patrones, clasificación de vehículos, método $k-N N$.

\section{Vehicle Recognition and Classification Using a Digital Accelerometer}

\begin{abstract}
Today, the pollution by urban is a growing problem in big cities. This work proposes making of analysis system of vibrations generate by automotive above urban avenues. By digital processing of signals using the envelope of signals for measure of vibrations. Is present a computational method that allows identifying various characteristics such as the magnitude, frequency and the kind of automotive implementing a classifier 1-NN. Experimental results show evidence of good functioning of the system, providing a tool that will allow start studies to depeer about the problems generated by vibrations on communication routs, as well as their effect on nerby buildings.
\end{abstract}

Keywords: accelerometer, vibrations, pattern recognition, vehicle classification, kNN method. 


\section{Introducción}

Estudios estadísticos se utilizan como una herramienta para la evaluación y generación de estrategias que agilicen o mejoren el flujo vehicular. La principal tarea de estos estudios es contar las frecuencias y tipo de vehículos que pasan por una vía [1]. Aunque pareciera una tarea simple contar vehículos, resulta una tarea tediosa y complicada si el flujo de vehículos es grande. Es por eso que se comenzaron a utilizar sensores de inducción, siendo estos los más usado desde los años 60 [1].

A lo largo de varios años, se han probado diferentes sensores para la detección de vehículos [2-4] y posteriormente se implementaron técnicas de inteligenciar artificial para la clasificación de vehículos $[1,5,6]$. Esto permite conocer con mayor detalle cada clase con respecto a los datos de diversos sensores de tráfico. Los sensores de tráfico pueden ser clasificados en dos tipos: invasivos y no invasivos. Estos últimos son instalados sobre la superficie de las vialidades, volviéndolos más populares y recurrentes, por su fácil mantenimiento [4].

El acelerómetro, el cual mide vibraciones mecánicas que el vehículo propaga por la superficie [6,5,7], se puede considerar como un sensor no invasivo. La vibración de un vehículo es distinta entre los diferentes tipos, ya que comúnmente el modelo muy característico, creados a base del estudio estocástico de las vibraciones generadas por los vehículos, para cada clase es distinto y por ende la vibración resulta diferente [8]. El éxito de esto sistemas ha llevado a incorporar otros tipos de sensores [6] o el aplicar compensaciones mediante estudios sísmicos anticipados [5]; sin embargo, esto limita la capacidad de los sensores de vibraciones y no permite exportarlos de manera adecuada.

Este trabajo se centra en la implementación de un acelerómetro digital MMA8451Q, encontrando sus límites y generalizando el proceso de evaluación con un solo sensor para el reconocimiento y clasificación de vehículos automotores. En este caso se encontrarán las diferencias entre un auto y una camioneta. Sobre estas muestras se aplica un clasificador $k$-NN ( $k$-Nearest Neighbor) para determinar la clase del vehículo, analizados mediante la ubicación de un solo umbral [9].

\section{Sobre las vibraciones y la clasificación de vehículos}

\subsection{Clasificación de vehículos}

La mayoría de las actividades humanas implican vibraciones en una u otra forma; por ejemplo, oímos porque nuestros tímpanos vibran y vemos por qué las ondas luminosas vibran. La respiración está asociada con la vibración de los pulmones y caminar implica el movimiento oscilatorio (periódico) de piernas y manos. El habla humana requiere el movimiento oscilatorio de la laringe y de la lengua. En años recientes, muchas aplicaciones de la vibración en el campo de la ingeniería han motivado a los investigadores, entre ellas el diseño de máquinas, cimientos, estructuras, motores, turbinas y sistemas de control [10].

Las vibraciones superficiales son una gran preocupación para los seres humanos, cuando estas vibraciones entran en los edificios, donde la mayor preocupación es que las vibraciones lleguen a afectar la estructura de los edificios, pero las oscilaciones son 
insuficientes para causar daños en la estructura; sin embargo, las vibraciones pueden afectar a las personas e incluso a muy bajo nivel pueden dañar la sensibilidad en equipos de laboratorio o afectar la manufactura de circuitos micro electrónicos [8].

Para determinar la clase de un vehículo de manera dinámica, se implementan sensores que puedan obtener las características del vehículo y dependiendo del enfoque se recaban diferentes tipos datos; por ejemplo, puede ser las dimensiones del vehículo mediante una cámara [11] para después aplicar una serie de filtros y técnicas de visión por computadora. También mediante la interrupción de la corriente mediante sensores de inducción, que registran la perturbación generada por el paso de un vehículo, apoyado por técnicas de inteligencia artificial para una clasificación más certera [1]. Un caso es el generado por la coalición de países nórdicos mediante el proyecto NorSIKT [2], donde se emplearon varios sensores para efectuar una clasificación de vehículos de forma más compleja resultando en 5 clases.

Con respecto al estudio de las vibraciones, la clasificación, ha implicado tener conciencia que se debe conocer el medio por el cual se propaga este fenómeno y utilizar métodos de inteligencia artificial suficientemente capaces para llegar a un resultado [5], o sustentar la información con datos de otros sensores involucrando también inteligencia artificial [6], siempre proporcionándole más información al sensor de vibraciones con más información.

\subsection{Clasificador k-NN}

El método de $\mathrm{k}$ vecinos más cercanos $(k-N N)$ es un método de clasificación supervisado, es uno de los algoritmos de clasificación más eficientes y a la vez más simples que existen; este algoritmo está basado en el enfoque de métricas y está fundado en la suposición de que los patrones cercanos entre si pertenecen a la misma clase y por ello un nuevo patrón a clasificar se determina la proximidad de este patrón por medio de alguna medida de similitud. Generalmente se usa la distancia euclidiana y se va calculando la distancia con respecto a los $\mathrm{n}$ patrones ya existentes dentro del conjunto fundamental. Se clasifica a la clase del patrón $k$ más cercano, donde $k$ es un entero positivo generalmente impar [9].

El algoritmo por seguir para $k=1$ es el siguiente:

1. Se escoge una métrica a utilizar (normalmente la Euclidiana).

2. Se calculan las distancias de un patrón $\mathbf{x}$ desconocido por clasificar, a cada uno de los patrones del conjunto fundamental.

3. Se obtiene la distancia mínima.

4. Se asigna al patrón $\mathbf{x}$ a la clase del patrón con la mínima distancia.

Cuando se usa una $k$ mayor a 1 , se sigue el mismo procedimiento antes descrito, tan solo que para asignar la clase del patrón a clasificar se usa la regla de mayoreo.

\section{Metodología y desarrollo}

\subsection{Sistema de medición de vibraciones}

Teniendo en cuenta que únicamente se usa un solo sensor, importa la forma en que el sistema se encuentra consolidado. 
El sistema básicamente se encuentra conformado por tres partes o dispositivos comunicados, como indica la Fig. 1.

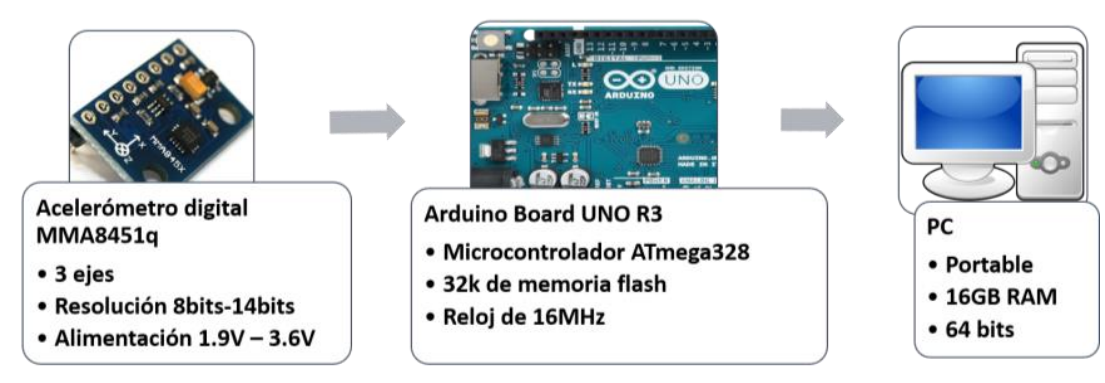

Fig. 1. Sistema de adquisición de datos en el orden como se obtienen los datos.

El acelerómetro digital MMA8451Q, tiene la capacidad de detectar las oscilaciones en los tres ejes fundamentales $x, y, z$; sin embargo, solo el eje $z$ mide las vibraciones producidas por los vehículos automotores a nivel superficial [7], por lo que serán tomados los datos de este eje, también maneja una resolución de 8 bits a 14 bits, permite un rango de salida de datos entre $1.56 \mathrm{~Hz}$ y $800 \mathrm{~Hz}$, utiliza el protocolo de comunicación $I^{2} C$ y tiene una escala dinámica de $\pm 2 g, \pm 4 g$ y $\pm 8 g$ [12]. La placa Arduino UNO [13], es implementado como un dispositivo de adquisición de los datos, debido a que ambos utilizan el mismo protocolo de comunicación $I^{2} C$ [14] lo que permite una rápida transferencia de datos. Siendo el Arduino el puente de comunicación entre el sensor y la Pc, este último es el medio por el cual se observarán los datos provenientes del acelerómetro

\subsection{Experimentación}

Para esta etapa experimental, se diseña una prueba que se repetirá durante múltiples ocasiones. Se utilizan dos vehículos de diferentes clases: una camioneta GMC Safari modelo 93 con un peso aproximado de 2.0 Ton y un Nissan Sentra modelo 2016 con un peso aproximado de 1.2 Ton. La velocidad promedio para el experimento es entre $20 \mathrm{~km} / \mathrm{h}$ y $30 \mathrm{~km} / \mathrm{h}$. En la Fig. 2 se indica la distancia en la cual la prueba será registrada, evitando la perdida de datos.

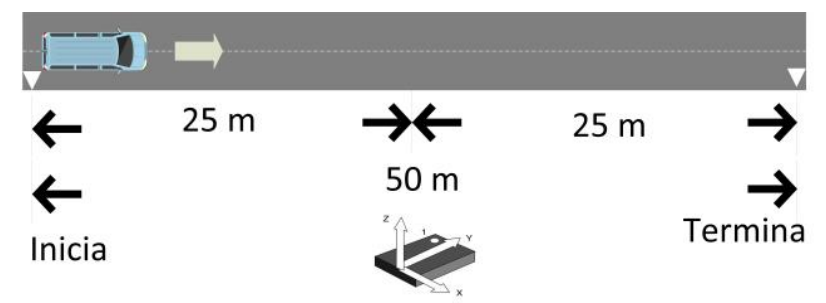

Fig. 2. Esquema que representa como se lleva a cabo una prueba. La distancia total para iniciar y terminar la medición de las vibraciones es de 50 metros, donde la disposición del acelerómetro es la mitad de esta distancia. 


\subsection{Metodología usada}

La cantidad de pruebas a efectuar para formar la base de datos en esta etapa experimental son 10 pruebas para cada uno de los vehículos. Para validar que la prueba fue adecuada, se cuenta con que el evento sea similar a la Fig. 3, donde la sección ovalada es la vibración producida al paso de un vehículo cerca del acelerómetro y las secciones dentro de los rectángulos son el comportamiento común del acelerómetro, por lo que estos datos se descartan, quedándonos solo con los datos contenidos dentro de la sección ovalada mostrado en la Fig. 3.

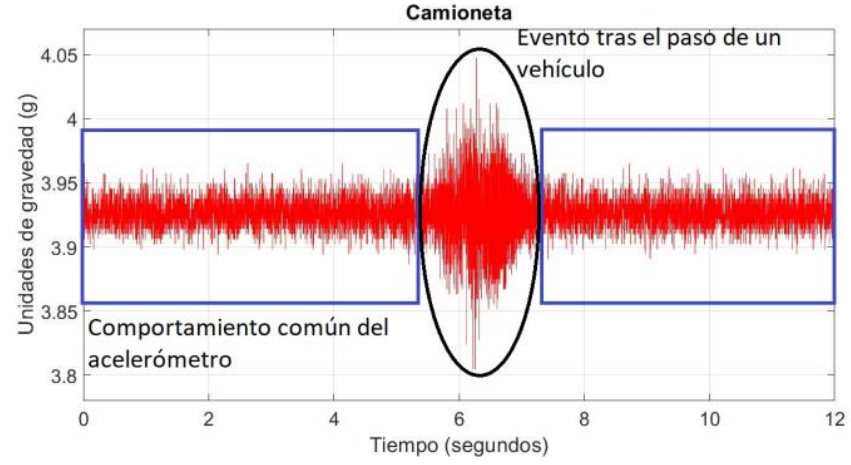

Fig. 3. Registro de un evento. Esta serie de datos implica dos partes, el comportamiento común del acelerómetro, y el evento tras el paso de un vehículo, en este caso un auto.

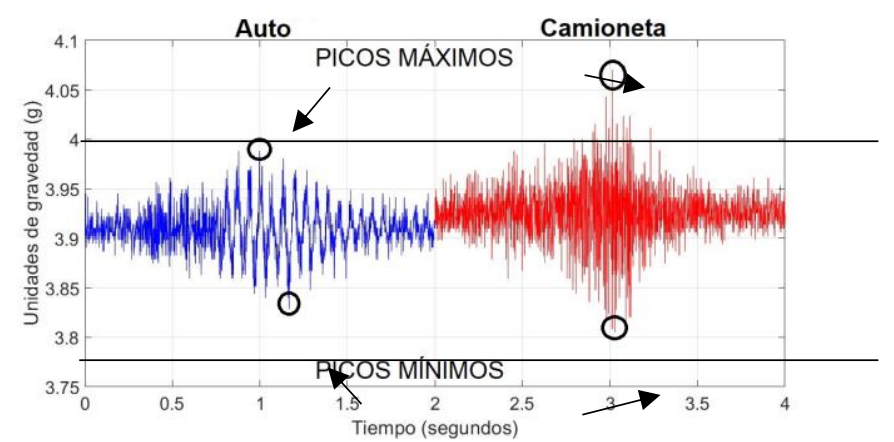

Fig. 4. Vibración producida por un auto (azul) comparada contra una camioneta (rojo), donde se indican los picos máximos (circulo superior) y los picos mínimos (circulo inferior).

Un método desarrollado para encontrar la separabilidad entre cada clase de vehículos es medir los picos máximos de cada prueba; estos se señalan en la Fig. 4. De esta manera se puede comprobar si los datos son factibles para integrar a la base de datos. Posteriormente para procesar los datos y generar un vector característico, se implementa un filtro digital FIR pasa banda descrita en (4) el cual emplea una banda de frecuencia de $100 \mathrm{~Hz}$ a $150 \mathrm{~Hz}$ e implementa el método de ventana, específicamente del tipo Hamming, este permite pasar las frecuencias características de las de las dos clases de vehículos, se determina el espectro de potencia elevando al cuadrado la señal filtrada y se encuentra la envolvente de los datos con (5) [15], el resultado para auto y 
camioneta se muestra en la Fig. 5 en la cual se puede apreciar que existe una notable diferencias entre las señales de las dos clases de vehículos.

$$
\begin{gathered}
h[n]=2 f_{a} \frac{\sin \left(n \omega_{a}\right)}{n \omega_{a}}-2 f_{b} \frac{\sin \left(n \omega_{b}\right)}{n \omega_{b}}, \\
w(n)=0.54-0.46 \cos \left(2 \pi \frac{n}{N}\right), 0 \leq n<N, \\
h_{d}[n]=w[n] h[n], \\
H_{d}(\Omega)=\sum_{n=0}^{N-1} h_{d}[n] e^{-j n \Omega}, \\
s[n]=\sum_{i=0}^{K-1} w[n-i]=\cdots=s[n-1]+w[n]-w[n-K] .
\end{gathered}
$$

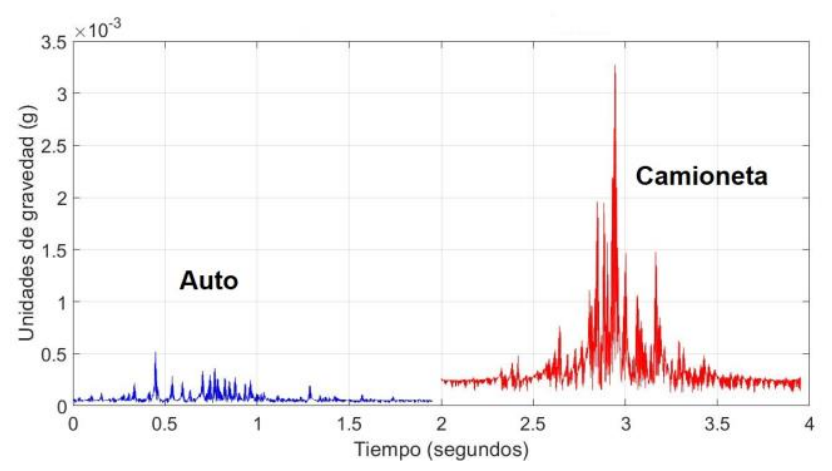

Fig. 5. Comparación entre la prueba de un auto contra una camioneta después de ser procesadas.

\subsection{Clasificador k-NN}

El clasificador usado es el $k$-NN, en su variante $1-N N$, que se enfoca en la clasificación con respecto a la distancia métrica más corta [9]. Como no involucra una retroalimentación, el tiempo de computo no es extenso; sin embargo, el 1-NN efectúa la evaluación sobre toda la base de datos. Por lo tanto, al implementar el clasificador, se utilizará el $100 \%$ del entrenamiento.

\section{Resultados y discusiones}

La extracción de los picos máximos (valores positivos) y mínimos (valores negativos) permiten no solo saber la amplitud de su mayor oscilación, también se puede determinar una separación lineal entre los vehículos de clases distintas. Esta separación se determina diferenciando los picos máximos y mínimos del vehículo de cierta clase con los de un vehículo de la clase contraria y así generando un umbral. De los dos picos determinados, el máximo puede ser suficiente para determinar esta separabilidad.

La reducción del tamaño de las muestras y el procesamiento de estas, nos permiten ver de manera gráfica en la Fig. 6, una mejor separabilidad, lo que implica que se podrán obtener mejores resultados en las clasificaciones, que es el objetivo de los distintos procesamientos sobre la señal. 
En la Tabla 1 (izquierda), se muestra el resultado de los porcentajes obtenidos de la clasificación realizada usando el 1-NN, en la cual se puede observar que se clasificaron bien ambas clases de vehículos. El 100\% acierto indica que las pruebas son adecuadas para el clasificador 1-NN para determinar la clase de nuevas muestras; sin embargo, para justificar esta afirmación se implementa el método de validación leave one out [16] cuyos resultados de clasificación son buenos dados las pocas pruebas de la base de datos, estos resultados también se muestran en la Tabla 1 (derecha), mostrados los resultados de esta forma, permite observar el comportamiento de las pruebas de dos maneras diferentes, obviando que las base de datos es adecuada para la clasificación de nuevas instancias para las dos clases de vehículos.
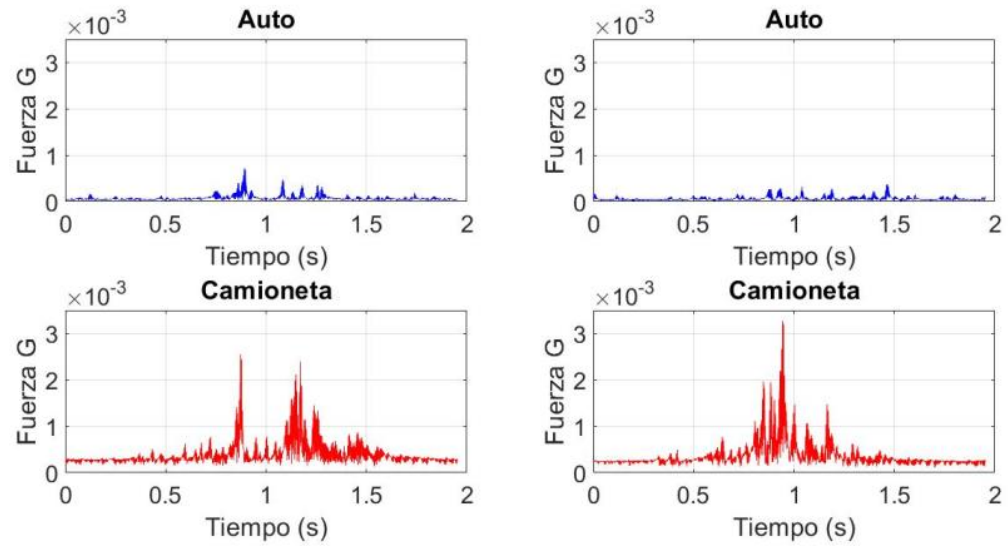

Fig. 6. Distintas señales de autos (azul) y de camionetas (rojo). Es el resultado del procesamiento de las señales, permitiendo obtener una mejor perspectiva de la separabilidad de los datos.

Tabla 1. Resultados de clasificación usando $1-\mathrm{NN}$ con entrenamiento del 100\% (izquierda) y usando validación leave one out (derecha).

Entrenamiento $100 \%$

Leave one out

\begin{tabular}{|r|r|r|c|}
\hline Clase de vehículo & $\begin{array}{c}\text { Porcentaje de } \\
\text { clasificación }\end{array}$ & Clase de vehículo & $\begin{array}{c}\text { Porcentaje de } \\
\text { clasificación }\end{array}$ \\
\hline Auto & $100 \%$ & Auto & $80 \%$ \\
\hline Camioneta & $100 \%$ & Camioneta & $80 \%$ \\
\hline
\end{tabular}

\section{Conclusiones}

El sistema de reconocimiento de vehículos resultó ser efectivo para medir el fenómeno de vibraciones comprobando su implementación. La efectiva clasificación de las pruebas actuales mediante el algoritmo implementado de clasificación permite identificar las clases de los vehículos y así determinar la clase a la que pertenecen, aunque se debe trabajar en incrementar el tamaño de la base de datos para hacer aún más efectiva la clasificación. Para obtener la mejor clasificación fueron fundamentales las técnicas de procesamiento de señales implementadas las cuales permitieron diferenciar de una manera más clara la característica de cada una de las clases. 


\section{Referencias}

1. Oliveira, H.A., Barbosa, F.R., Almeida, O.M., Braga, A.P.S.: A vehicle classification based on inductive loop detectors using artificial neural networks. In: 2010 9th IEEE/IAS International Conference on Industry Applications - INDUSCON 2010. pp. 1-6 (2010)

2. NorSIKT-Nordic System for Intelligent Classification of Traffic. Procedia - Soc. Behav. Sci. 48, 1702-1712 (2012)

3. Traffic Detector Handbook. Federal Highway Administration (2006)

4. Elena Mimbela Project Manager, L.Y., Klein, L.A., Kent, P., Project Consultant John Hamrick, V.L., Project Consultant Karen Luces, V.M., Sylvia Herrera, N.: Summary Of Vehicle Detection And Surveillance Technologies Used in Intelligent Transportation Systems Submitted To: Federal Highway Administration $\mathrm{s}$ (FHWA) Intelligent Transportation Systems Joint Program Office Program Office. (2000)

5. Lan, J., Lan, T., Nahavandi, S.: A Novel Application of a Microaccelerometer for Target Classification. IEEE Sens. J. 4, 519-524 (2004)

6. Kleyko, D., Hostettler, R., Birk, W., Osipov, E.: Comparison of Machine Learning Techniques for Vehicle Classification Using Road Side Sensors. In: 2015 IEEE 18th International Conference on Intelligent Transportation Systems. pp. 572-577 (2015)

7. Hostettler, R., Birk, W., Lundberg Nordenvaad, M.: Feasibility of road vibrations-based vehicle property sensing. IET Intell. Transp. Syst. 4, 356 (2010)

8. Hunt, H.E.M.: Stochastic modelling of traffic-induced ground vibration. J. Sound Vib. 144, 53-70 (1991)

9. Hernández-Bautista, I., Carrasco-Ochoa, J.A., Martínez-Trinidad, J.F., Camacho-Nieto, O., Pogrebnyak, O.: Adjustment of Wavelet Filters for Image Compression Using Artificial Intelligence. Polibits 53, 23-30 (2016)

10. Rao, S.S.: Vibraciones mecánicas. Pearson Educación (2012)

11. Gupte, S., Masoud, O., Martin, R.F.K., Papanikolopoulos, N.P.: Detection and classification of vehicles. IEEE Trans. Intell. Transp. Syst. 3, 37-47 (2002)

12. Semiconductors, N.: MMA8451Q 3-Axis, 14-bit/8-bit Digital Accelerometer, Data sheet.

13. Arduino Uno Rev3, https://store.arduino.cc/usa/arduino-uno-rev3

14. AN10216-01 I Integrated Circuits 2 C Manual Jean-Marc Irazabal - I Steve Blozis - I. (2003)

15. Hostettler, R.: Master's Thesis Traffic Counting Using Measurements of Road Surface Vibrations. (2009)

16. Shao, Z., Er, M.J., Wang, N.: An Efficient Leave-One-Out Cross-Validation-Based Extreme Learning Machine (ELOO-ELM) With Minimal User Intervention. IEEE Trans. Cybern. 46, 1939-1951 (2016) 